\title{
Genetic heterogeneity of patients with suspected Silver-Russell syndrome: genome-wide copy number analysis in 82 patients without imprinting defects
}

Takanobu Inoue ${ }^{1,2}$, Akie Nakamura', Tomoko Fuke' , Kazuki Yamazawa', Shinichiro Sano', Keiko Matsubara', Seiji Mizuno ${ }^{3}$, Yoshika Matsukura ${ }^{4}$, Chie Harashima ${ }^{4}$, Tatsuji Hasegawa ${ }^{5}$, Hisakazu Nakajima ${ }^{5}$, Kumi Tsumura ${ }^{6}$, Zenro Kizaki ${ }^{7}$, Akira Oka ${ }^{2}$, Tsutomu Ogata $^{1,8}$, Maki Fukami ${ }^{1}$ and Masayo Kagami ${ }^{{ }^{*}}$

\begin{abstract}
Background: Silver-Russell syndrome (SRS) is a rare congenital disorder characterized by pre- and postnatal growth failure and dysmorphic features. Recently, pathogenic copy number variations (PCNVs) and imprinting defects other than hypomethylation of the H19-differentially methylated region (DMR) and maternal uniparental disomy chromosome 7 have been reported in patients with the SRS phenotype. This study aimed to clarify the frequency and clinical features of patients with SRS phenotype caused by PCNVs.

Methods: We performed array comparative genomic hybridization analysis using a catalog array for 54 patients satisfying the Netchine-Harbison clinical scoring system (NH-CSS) (SRS-compatible) and for 28 patients presenting with three NH-CSS items together with triangular face and/or fifth finger clinodactyly and/or brachydactyly (SRSlike) without abnormal methylation levels of 9 DMRs related to known imprinting disorders. We then investigated the clinical features of patients with PCNVs.

Results: Three of the 54 SRS-compatible patients (5.6\%) and 2 of the 28 SRS-like patients (7.1\%) had PCNVs. We detected $3.5 \mathrm{Mb}$ deletion in 4p16.3, mosaic trisomy 18, and 3.77-4.00 Mb deletion in 19q13.11-12 in SRS-compatible patients, and 1.41-1.97 Mb deletion in 7q11.23 in both SRS-like patients. Congenital heart diseases (CHDs) were identified in two patients and moderate to severe global developmental delay was observed in four patients.

Conclusions: Of the patients in our study, 5.6\% of SRS-compatible and $7.1 \%$ of SRS-like patients had PCNVs. All PCNVs have been previously reported for genetic causes of contiguous deletion syndromes or mosaic trisomy 18. Our study suggests patients with PCNVs, who have a phenotype resembling SRS, show a high tendency towards CHDs and/or apparent developmental delay.
\end{abstract}

Keywords: Silver-Russell syndrome, Pathogenic copy number variation, Array comparative genomic hybridization, Netchine-Harbison clinical scoring system, 4p microdeletion syndrome, Mosaic trisomy 18, 19q13.11 deletion syndrome, Williams syndrome

\footnotetext{
* Correspondence: kagami-ms@ncchd.go.jp

${ }^{1}$ Department of Molecular Endocrinology, National Research Institute for

Child Health and Development, 2-10-1, Okura Setagaya-ku, Tokyo 157-8535,

Japan

Full list of author information is available at the end of the article
} 


\section{Findings}

\section{Background}

Silver-Russell syndrome (SRS) is a rare congenital disorder characterized by pre- and postnatal growth failure and dysmorphic features [1]. The diagnosis of SRS is based on a combination of clinical features. Several clinical scoring systems for SRS have been proposed [2-8]. Of these, the Netchine-Harbison clinical scoring system (NH-CSS) is the most sensitive and has the highest negative predictive value [8]. NH-CSS includes six items: (1) small for gestational age (birth weight and/or birth length $\leq-2$ standard deviation score (SDS) for gestational age); (2) postnatal growth retardation (height at $24 \pm 1$ months $\leq-2$ SDS or height $\leq-2$ SDS below midparental target height); (3) relative macrocephaly at birth (head circumference at birth $\geq 1.5$ SDS above birth weight and/or length SDS); (4) protruding forehead (forehead projecting beyond the facial plane from the side view among toddlers); (5) body asymmetry (leg length discrepancy (LLD) of $\geq 0.5 \mathrm{~cm}$ or arm asymmetry or LLD $<0.5 \mathrm{~cm}$ with at least two other asymmetrical body parts (one non-face)); (6) feeding difficulties and/or low body mass index (BMI) (BMI $\leq-2$ SDS at 24 months or current use of a feeding tube or cyproheptadine for appetite stimulation). When patients meet four or more of the six NH-CSS items, they are clinically diagnosed with SRS [8]. Furthermore, additional clinical features such as triangular face, fifth finger clinodactyly, and brachydactyly were frequently identified in SRS patients [9], but these clinical features are not included in the $\mathrm{NH}^{-}$ CSS items.

The most common genetic causes of SRS are hypomethylation of the H19-differentially methylated region (DMR) at the 11p15 imprinted region (H19-hypo) and maternal uniparental disomy chromosome 7 (UPD(7)mat) [1]. Recently, Azzi et al. reported that H19hypo and UPD(7)mat were detected in 58.3 and $18.3 \%$ of patients, respectively, satisfying the NH-CSS criteria [8]. In addition, $11 \mathrm{p} 15$ duplication and other imprinting disorders such as Temple syndrome, maternal uniparental disomy for chromosome 16 and 20, and pathogenic copy number variations (PCNVs) are less frequently identified in patients with SRS clinical phenotypes $[8,10]$.

Several reports have described patients with the SRS phenotype caused by PCNVs, but these reports have the following weak points: (1) insufficient clinical information on the patients; (2) imprinting disorders other than SRS were not excluded, and (3) the number of patients involved in these studies was small $[6,8,11-13]$. To clarify the frequency and the clinical features of patients with the SRS phenotype caused by PCNVs, we performed molecular and clinical studies in patients with suspected SRS who had normal methylation levels for 9 DMRs related to known imprinting disorders.

\section{Methods \\ Patients}

We summarized the inclusion criteria in Fig. 1. We studied 82 out of 292 patients referred to us for genetic diagnosis of SRS from 2002 to 2016. All 82 patients had normal methylation levels for $9 \mathrm{DMRs}$ related to known imprinting disorders, that is, H19-DMR, PEG1/MESTDMR, PEG10-DMR, PLAGL1-DMR, KCNQ1OT1-DMR, IG-DMR, MEG3-DMR, SNRPN-DMR, and GNAS-A/BDMR. Methylation analysis was performed by combined bisulfite restriction analysis or pyrosequencing as previously reported $[9,14]$. Fifty-five out of 82 patients were examined using karyotyping before being referred to us and were confirmed to have normal karyotypes. All patients were Japanese except for two patients who were from Canada and the USA.

We collected clinical information from the attending physicians by questionnaire. Fifty-four patients who satisfied $\geq 4$ out of $6 \mathrm{NH}$-CSS criteria were included in this study and were classified as SRS-compatible. Furthermore, because some patients who did not satisfy NHCSS criteria had H19-hypo or UPD(7)mat [8, 15-17], 28 patients who presented three NH-CSS items together with triangular face and/or fifth finger clinodactyly and/ or brachydactyly, which are common clinical features in SRS patients [9], were also included in this study and were classified as SRS-like. For patients under 23 months old, the score for postnatal growth retardation was excluded from the NH-CSS criteria. When these patients satisfied four or five NH-CSS items or three NH-CSS items together with triangular face and/or fifth finger clinodactyly and/or brachydactyly, we classified them as SRS-compatible or SRS-like, respectively.

\section{Molecular studies}

We extracted leucocyte genomic DNA from peripheral blood using the Gentra Puregene Blood Kit (Qiagen, Hilden, Germany). We then performed array comparative genomic hybridization analysis using a $60 \mathrm{~K}$ catalog array (Agilent Technologies, Palo Alto, CA, USA) in 82 patients with normal methylation levels in 9 DMRs. To detect PCNVs, we first extracted CNVs that had three or more continuous probes with aberrant signals. Next, we evaluated whether these CNVs were benign or pathogenic. In our study, the CNVs reported in the Database of Genomic Variants [18] as a normal variant were considered to be nonpathogenic, while CNVs that have been previously reported to cause some of the known syndromes were considered to be pathogenic. In cases where CNVs were not reported in the database or medical publications, we searched whether the genes involved in the regions were associated with the SRS phenotype. Karyotyping was performed when necessary. 


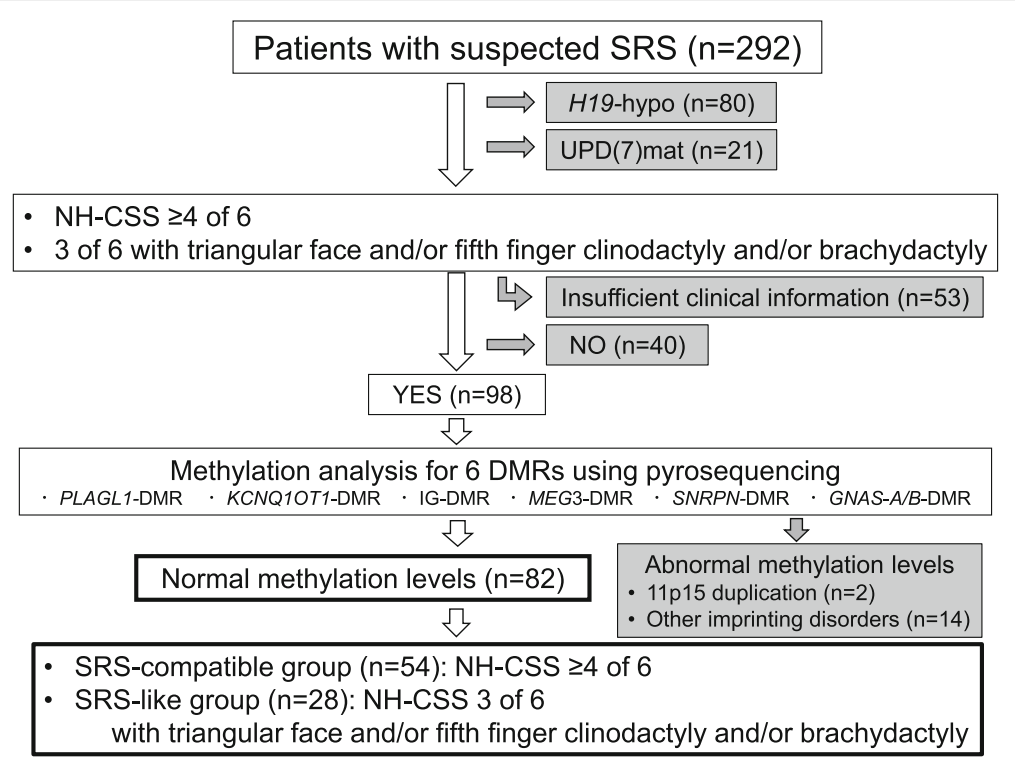

Fig. 1 The flowchart of inclusion criteria. From 2002 to 2016, 292 patients were referred to us for genetic diagnosis of Silver-Russell syndrome (SRS). We studied 82 patients with SRS clinical features, and no abnormal methylation levels for 9 differentially methylated regions related to known imprinting disorders.

\section{Clinical studies}

We compared the clinical features between our patients with PCNVs and patients with genetic syndromes caused by the same PCNVs. Furthermore, we calculated the frequencies of congenital heart diseases (CHDs), motor developmental delay, and speech delay in patients with PCNVs. Echocardiography and tests for intelligence quotient or developmental quotient were performed in all patients with PCNVs.

\section{Statistical analysis}

The frequencies of clinical feature differences between patients with PCNVs, H19-hypo, and UPD(7)mat were analyzed by Fisher's exact probability test using a statistical software program (StatFlex for Windows Ver.6.0, Artec, Osaka, Japan). $P<0.05$ was considered significant.

\section{Results}

\section{Molecular studies}

We identified 3 patients (patients 1-3) with PCNVs from 54 patients in the SRS-compatible group (5.6\%), and 2 patients (patients 4 and 5) with PCNVs from 28 patients in the SRS-like group (7.1\%) (Table 1). We detected $3.5 \mathrm{Mb}$ deletion in $4 \mathrm{p} 16.3$ including the critical region of $4 \mathrm{p}$ microdeletion syndrome in patient 1 [19] (Fig. 2a), trisomy 18 in patient 2 (Fig. 2b) and 3.77$4.00 \mathrm{Mb}$ deletion in 19q13.11-12 involving the smallest region of overlap of 19q13.11 deletion syndrome [20] in patient 3 (Fig. 2c). Subsequently, we carried out karyotyping using peripheral blood and ascertained mosaic trisomy $18(46, \mathrm{XY}[14] / 47, \mathrm{XY},+18[6])$ in patient 2 . In the SRS-like group, we detected the same copy number change (1.41-1.97 Mb deletion in 7q11.23), which included the critical region of Williams syndrome [21] in patients 4 and 5 (Fig. $2 \mathrm{~d}$ for patient 4 , 2e for patient 5). Both parents of patients $1,3,4$, and 5 did not present with the PCNVs detected in their children.

\section{Clinical studies}

We summarized clinical features of patients with PCNVs, which are included in NH-CSS items and characteristic of each genetic syndrome caused by each PCNV, in Table 1. Patient 1 with $3.5 \mathrm{Mb}$ deletion in 4 p16.3 was born at 34 weeks of gestation with severe neonatal asphyxia and periventricular leukomalacia. This girl had four of the NH-CSS items, which are also identified in 4p microdeletion syndrome [19]. She had the characteristic features of $4 \mathrm{p}$ microdeletion syndrome such as Greek warrior helmet appearance, severe global developmental delay, an atrial septal defect, epilepsy, and hearing loss [19]. Patient 2 with mosaic trisomy 18 was 1-year-and-9-month-old boy, so we could therefore not evaluate his postnatal growth retardation using the $\mathrm{NH}$-CSS. He presented with five other $\mathrm{NH}$-CSS items and a ventricular septal defect, however, he did not show overlapping fingers and rocker bottom feet, which are characteristic features of trisomy 18 [22]. Patient 3 with 19q13.11-12 deletion had four NH-CSS items and some of the characteristic features of 19q13.11 deletion syndrome such as cutis aplasia over the posterior occiput [20] (Fig. 3a). This patient presented with moderate global developmental delay. Patients 4 and 5 exhibited 


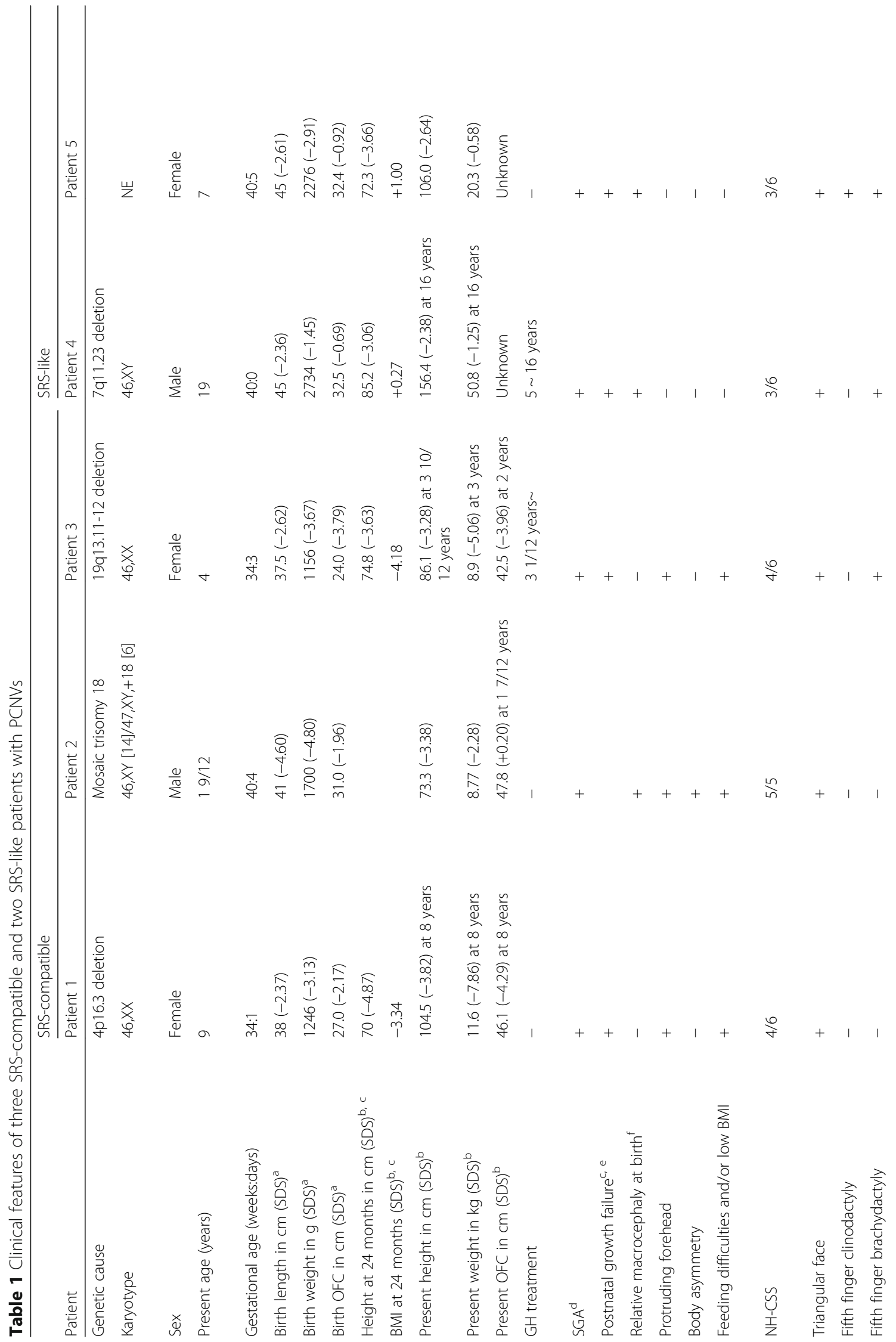




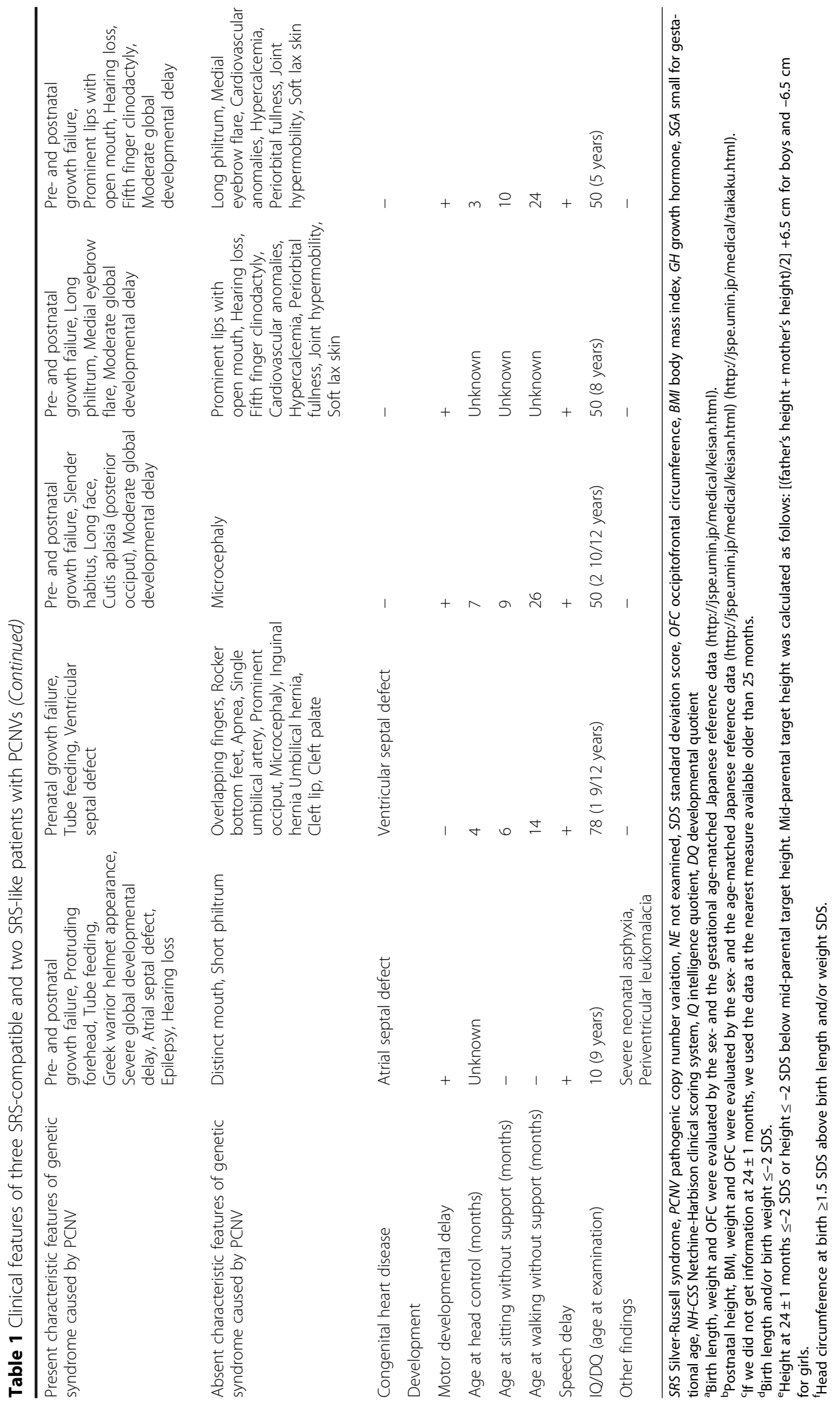




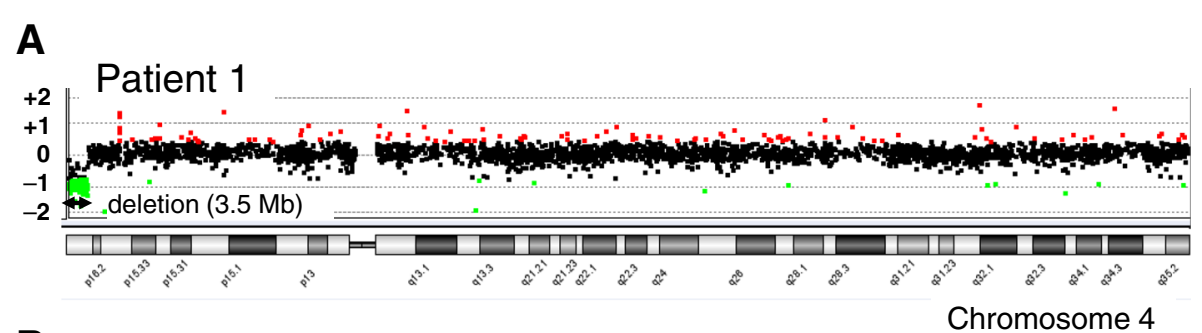

\section{B}

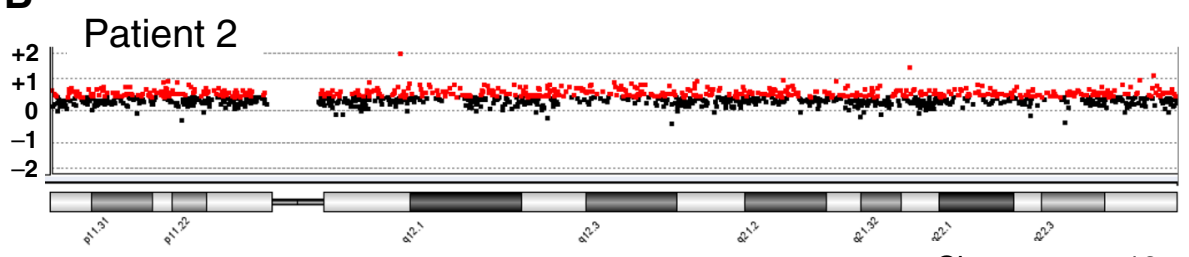

C

Chromosome 18

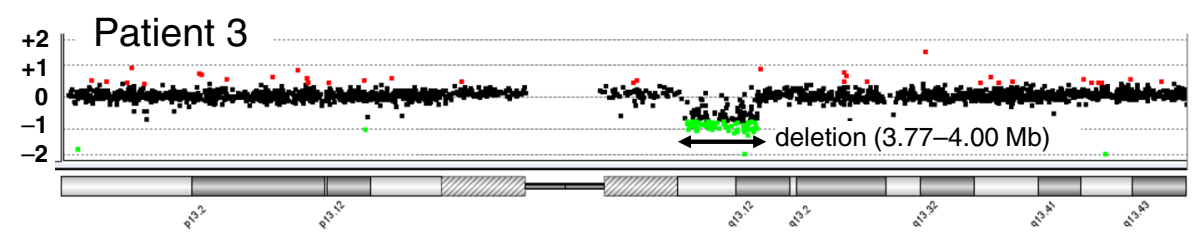

D

Chromosome 19

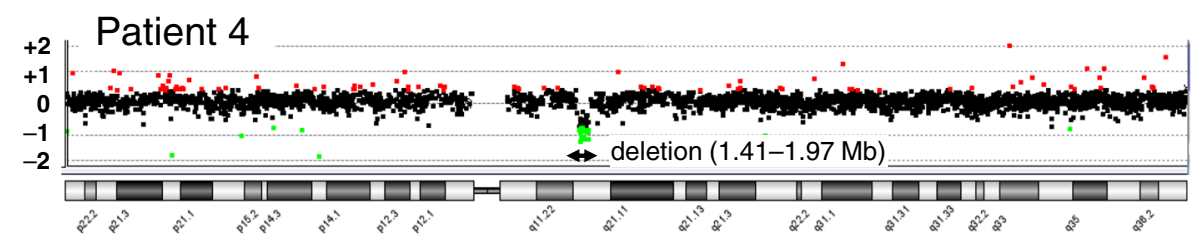

E

Chromosome 7

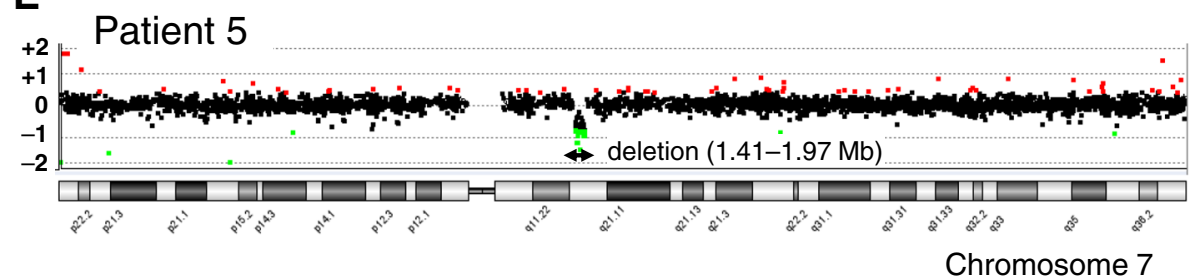

Fig. 2 Array comparative genomic hybridization profiles of the five patients with pathogenic copy number variations. a Patient 1 (SRS-compatible, 4p16.3 deletion). b Patient 2 (SRS-compatible, mosaic trisomy 18). c Patient 3 (SRS-compatible, 19q13.11-12 deletion). d Patient 4 (SRS-like, 7q11.23 deletion). e Patient 5 (SRS-like, 7q11.23 deletion). The black, red, and green dots denote signals indicative of the normal, increased (>+0.4), and decreased $(<-0.8)$ copy numbers, respectively

triangular face and fifth finger brachydactyly and/or clinodactyly together with three NH-CSS items and moderate global developmental delay. However, they did not present many of the characteristic findings of Williams syndrome such as cardiovascular anomalies, hypercalcemia, and distinctive facial features [23] (Fig. 3b for patient 4). The attending physicians of all patients with PCNVs other than patient 1 were not clinical geneticists.

The frequencies of the clinical features in patients with PCNVs are shown in Table 2. CHDs were identified in two patients. Motor developmental delay and speech delay were observed in almost all patients with PCNVs.

\section{Discussion}

We performed the largest genome-wide copy number analysis in 54 SRS-compatible patients and 28 SRS-like patients who had normal methylation levels for 9 DMRs related to imprinting disorders. PCNVs were identified in $5.6 \%$ of SRS-compatible patients and 7.1\% of SRS-like patients. There are two studies about genome-wide copy 


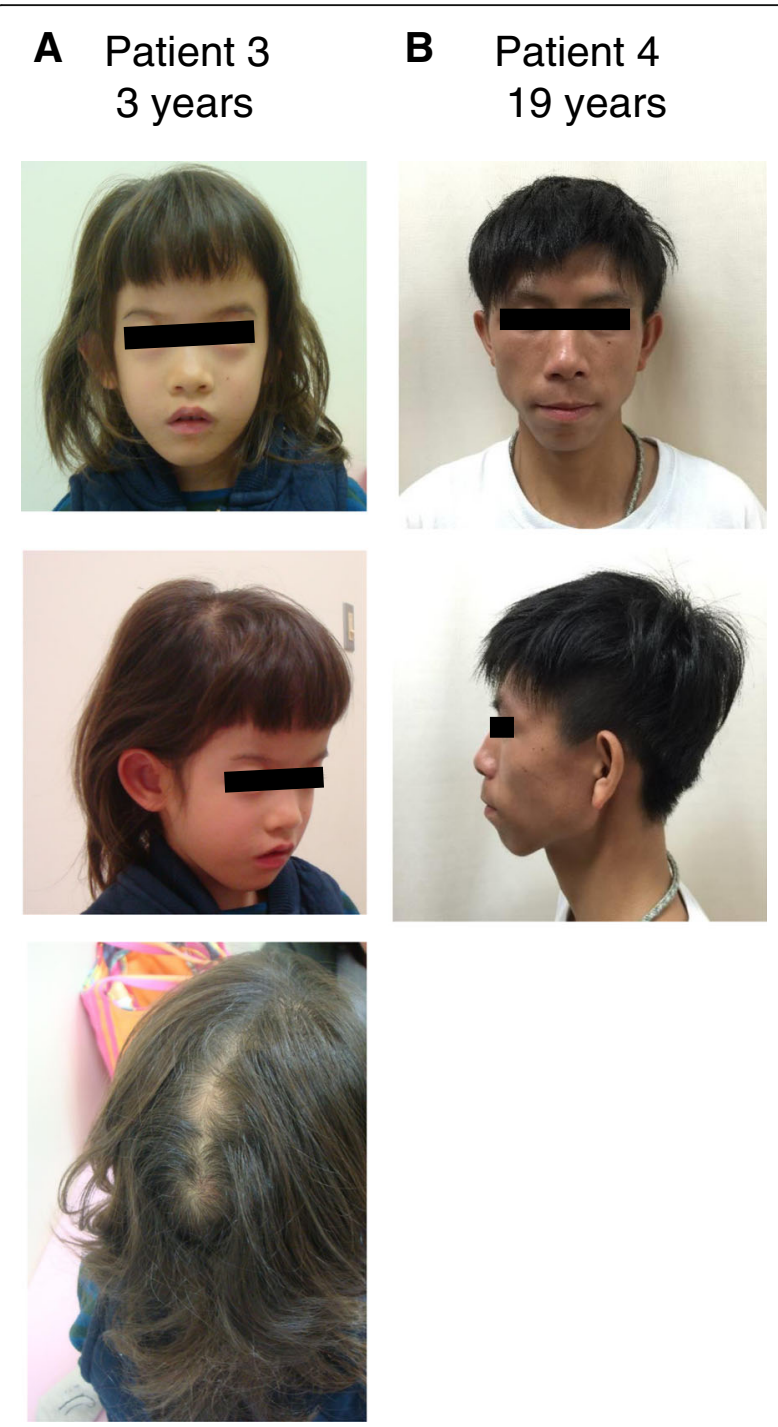

Fig. 3 Photographs of patients with pathogenic copy number variations. a Patient 3 (SRS-compatible, 19q13.11 deletion syndrome). The patient had cutis aplasia over the occiput, which is characteristic of 19q13.11 deletion syndrome. b Patient 4 (SRS-like, Williams syndrome)

number analysis in patients diagnosed as SRS using the $\mathrm{NH}-\mathrm{CSS}$. Azzi et al. identified 1 patient (9\%) with PCNV (1q21 microdeletion) in 11 patients with 4 or more $\mathrm{NH}$ CSS items who had neither H19-hypo, UPD(7)mat, CDKN1C mutation, imprinting abnormalities in other imprinted regions nor uniparental isodisomy other than UPD(7)mat [8]. Sachwitz et al. reported 2 patients (12\%) with PCNVs (5q35 duplication and 4p16.3 deletion together with $7 \mathrm{q} 36$ duplication) in 17 patients satisfying $\mathrm{NH}$-CSS criteria without H19-hypo, UPD(7)mat, gene mutations of CDKN1C and IGF2, and abnormal methylation levels in the 6q24, 14q32, and 20q13 imprinted regions [13]. Although there were differences in methods of molecular analysis and the number of the patients between our study and previous studies, the frequency of PCNVs in patients presenting the SRS phenotype without H19-hypo, UPD(7)mat and other imprinting disorders was similar among these studies.

PCNVs detected in this study have been previously reported to be responsible for $4 \mathrm{p}$ microdeletion syndrome, trisomy 18, 19q13.11 deletion syndrome, and Williams syndrome. To our knowledge, patients with suspected SRS have not been reported among patients with $4 \mathrm{p}$ microdeletion syndrome, 19q13.11 deletion syndrome, and Williams syndrome. A single case of mosaic trisomy 18 with suspected SRS had been previously reported [24]. This patient presented pre- and postnatal growth failure, poor sucking ability, slow weight gain, a relatively large head, body asymmetry of the chest and extremities, together with triangular face and fifth finger brachydactyly. Some NH-CSS items such as small for gestational age and feeding difficulties are also observed in patients with trisomy 18 [22]. Additionally, the clinical phenotype of mosaic trisomy 18 varies from the typical trisomy 18 phenotype to near-normal [25]. Actually, patient 2's phenotype was not typical for trisomy 18, but this patient had some common overlapping features between SRS and trisomy 18. Thus, mosaic trisomy 18 patients may present with SRS clinical features. Some clinical features of $4 \mathrm{p}$ microdeletion syndrome overlap with SRS phenotype: both SRS and 4p microdeletion syndrome have pre- and postnatal growth failure, some facial features such as a protruding forehead [19]. Severe developmental delay, epilepsy, and hearing loss are characteristic of $4 \mathrm{p}$ microdeletion syndrome [19]. Because patient 1 presented with the latter three symptoms and also experienced severe neonatal asphyxia and neurological sequelae, the attending physician who was a clinical geneticist thought that the patient had SRS and neurological problems caused by asphyxia. These findings show the difficulties in clinically diagnosing SRS in patients with severe neurological complications. To our knowledge, the 19q13.11 deletion syndrome identified in patient 3 has been reported in only 25 patients and presents with pre- and postnatal growth retardation, slender habitus, severe postnatal feeding difficulties, microcephaly, motor- and intellectual developmental delay, hypospadias, and cutis aplasia over the posterior occiput [20, 26-28]. Because phenotypic overlaps exist between $19 q 13.11$ deletion syndrome and SRS, we need to consider $19 q 13.11$ deletion syndrome as a differential diagnosis of SRS. Patients 4 and 5 presented atypical clinical features of Williams syndrome. In addition, pre- and postnatal growth failure, fifth finger clinodactyly, which were detected in patients 4 and/or 5 , are common clinical features between Williams syndrome and SRS [23]. Thus, Williams syndrome patients with atypical clinical features may be misdiagnosed with SRS. 
Table 2 Phenotypical comparison between patients with PCNVs and H19-hypo or UPD(7)mat

\begin{tabular}{|c|c|c|c|c|c|c|c|c|c|}
\hline & Patients with & CNVs in th & is study & Previous repor & & & & $P$ value & \\
\hline & $\begin{array}{l}\text { SRS- } \\
\text { compatible }\end{array}$ & SRS-like & Total & H19-hypo & & UPD(7)mat & & $\begin{array}{l}\text { PCNV vs. } \\
\text { H19-hypo }\end{array}$ & $\begin{array}{l}\text { PCNV vs. } \\
\text { UPD(7)mat }\end{array}$ \\
\hline$\overline{S G A^{a}}$ & $3 / 3(100 \%)$ & $\begin{array}{l}2 / 2 \\
(100 \%)\end{array}$ & $\begin{array}{l}5 / 5 \\
(100 \%)\end{array}$ & $43 / 43(100 \%)$ & & 9/9 (100\%) & & 1.0000 & 1.0000 \\
\hline Postnatal growth failure $e^{b, c}$ & $2 / 2(100 \%)$ & $\begin{array}{l}2 / 2 \\
(100 \%)\end{array}$ & $\begin{array}{l}4 / 4 \\
(100 \%)\end{array}$ & 29/35 (83\%) & & 8/9 (89\%) & & 0.6020 & 1.0000 \\
\hline $\begin{array}{l}\text { Relative macrocephaly at } \\
\text { birth }^{d}\end{array}$ & $1 / 3(33 \%)$ & $\begin{array}{l}2 / 2 \\
(100 \%)\end{array}$ & $\begin{array}{l}3 / 5 \\
(60 \%)\end{array}$ & 29/29 (100\%) & & 7/9 (78\%) & & 0.0178 & 0.5804 \\
\hline Protruding forehead & $3 / 3(100 \%)$ & 0/2 (0\%) & $\begin{array}{l}3 / 5 \\
(60 \%)\end{array}$ & $31 / 37(84 \%)$ & & $7 / 9(78 \%)$ & & 0.2368 & 0.5804 \\
\hline Body asymmetry & $1 / 3(33 \%)$ & 0/2 (0\%) & $\begin{array}{l}1 / 5 \\
(20 \%)\end{array}$ & $30 / 37(81 \%)$ & & $3 / 9(33 \%)$ & & 0.0126 & 1.0000 \\
\hline $\begin{array}{l}\text { Feeding difficulties and/or } \\
\text { low BMl }\end{array}$ & $3 / 3(100 \%)$ & 0/2 (0\%) & $\begin{array}{l}3 / 5 \\
(60 \%)\end{array}$ & $16 / 34(47 \%)$ & & 6/9 (67\%) & & 0.6614 & 1.0000 \\
\hline Congenital heart disease & $2 / 3(67 \%)$ & 0/2 (0\%) & $\begin{array}{l}2 / 5 \\
(40 \%)\end{array}$ & & $8 / 145(6 \%)^{\mathrm{e}}$ & & $0 / 17(0 \%)^{\mathrm{e}}$ & 0.0361 & 0.0433 \\
\hline Motor developmental delay & $2 / 3(67 \%)$ & $\begin{array}{l}2 / 2 \\
(100 \%)\end{array}$ & $\begin{array}{l}4 / 5 \\
(80 \%)\end{array}$ & $18 / 37(49 \%)^{\mathrm{e}}$ & & $6 / 9(67 \%)^{e}$ & & 0.3465 & 1.0000 \\
\hline Speech delay & $3 / 3(100 \%)$ & $\begin{array}{l}2 / 2 \\
(100 \%)\end{array}$ & $\begin{array}{l}5 / 5 \\
(100 \%)\end{array}$ & $8 / 31(26 \%)^{\mathrm{e}}$ & & $6 / 9(67 \%)^{\mathrm{e}}$ & & 0.0034 & 0.2582 \\
\hline Reference & & & & $\begin{array}{l}\text { Fuke T, et al. } \\
{[9]}\end{array}$ & $\begin{array}{l}\text { Ghanim M, et al. } \\
\text { [29] }\end{array}$ & $\begin{array}{l}\text { Fuke } T \text {, et al. } \\
{[9]}\end{array}$ & $\begin{array}{l}\text { Ghanim M, et al. } \\
\text { [29] }\end{array}$ & & \\
\hline
\end{tabular}

PCNV pathogenic copy number variation, H19-hypo hypomethylation of the H19-differentially methylated region at the $11 \mathrm{p} 15$ imprinted region, UPD(7)mat maternal uniparental disomy chromosome 7, SRS Silver-Russell syndrome, SGA small for gestational age, BMI body mass index

airth length and/or birth weight $\leq-2$ standard deviation score (SDS) of the sex- and the gestational age-matched Japanese reference data (http://jspe.umin.jp/medical/keisan.html)

${ }^{b}$ Height at $24 \pm 1$ months $\leq-2$ SDS of the sex- and the age-matched Japanese reference data (http://jspe.umin.jp/medical/keisan.html)

'If we did not get information at $24 \pm 1$ months, we used the data at the nearest measure available older than 25 months

${ }^{\mathrm{d}}$ Head circumference at birth $\geq 1.5$ SDS above birth length and/or weight SDS

${ }^{\mathrm{e}}$ It is not clear that echocardiography and tests for intelligence quotient or developmental quotient were performed in all patients in these previous reports Significant $P$ values $(<0.05)$ are italicized

We compared the frequencies of the clinical features between patients with PCNVs and previously reported patients with H19-hypo or UPD(7)mat [9, 29] (Table 2). The frequency of CHD was significantly higher in patients with PCNVs than in both patients with H19-hypo and patients with UPD(7)mat. Regarding the frequency of motor development, significant differences were not identified between two groups (PCNVs vs. H19-hypo and PCNVs vs. UPD(7)mat). The incidence of speech delay in patients with PCNVs was significantly higher than that in patients with H19-hypo, and there was no significance between patients with $\mathrm{PCNV}$ s and those with UPD(7)mat. In patients with UPD(7)mat, abnormal gene expression of FOXP2 on chromosome 7 associated with language development may lead to speech delay [30]. All of the patients with PCNVs other than patient 2 presented moderate to severe global developmental delay, while most of the patients with H19-hypo and UPD(7)mat in a previous study presented with mild delay or normal range [16]. Our study suggests that patients with PCNVs, who have a phenotype resembling SRS, have a high tendency towards CHD or apparent developmental delay. To provide better treatment and genetic counseling, we suggest that attending physicians perform copy number analysis prior to methylation analysis for patients with the SRS phenotype together with CHD, apparent developmental delay, or other dysmorphic features.

Our study has some limitations. First, as several attending physicians including general pediatricians, neonatologists, pediatric endocrinologists, and pediatric geneticists, assessed patients' clinical features, diagnostic bias may have occurred during clinical diagnosis for those items that were subjectively diagnosed, such as a protruding forehead, triangular face, fifth finger clinodactyly, and brachydactyly. In addition, since all attending physicians were not specialists in human genetics, clinical diagnoses for genetic syndromes may not be accurate. Second, although the NH-CSS is the most sensitive and has the highest negative predictive value among previously reported SRS clinical scoring systems, because most clinical features of SRS are not specific, the specificity of $\mathrm{NH}$-CSS is low (36\%), which is similar to that of other scoring systems [8]. As clinical diagnosis based on only NH-CSS might result in misdiagnosis with SRS, the evaluation of the clinical features other than 
$\mathrm{NH}$-CSS items is also important for diagnosis. Ideally, the patients with the SRS phenotype should be evaluated by clinical geneticists prior to performing genetic testing.

In conclusion, $5.6 \%$ of SRS-compatible and 7.1\% of SRSlike patients had PCNVs in our study. In addition, our study revealed the difficulties of diagnosing SRS in patients with severe neurological complications, and the need to consider mosaic trisomy 18, 19q13.11 deletion syndrome and atypical Williams syndrome for a differential diagnosis of SRS. Patients misdiagnosed with SRS who have PCNVs may have a high tendency towards CHDs and/or apparent developmental delay. To provide better treatment and genetic counseling for patients with the SRS phenotype together with CHD, apparent developmental delay, or other dysmorphic features, attending physicians should consider the alternative diagnoses or refer patients to clinical geneticists prior to performing genetic testing. Furthermore, if molecular analysis is required, copy number analysis should be considered before methylation analysis.

\section{Abbreviations \\ BMI: Body mass index; CHD: Congenital heart disease; DMR: Differentially methylated region; H19-hypo: Hypomethylation of the H19-DMR at the 11 p15 imprinted region; LLD: Leg length discrepancy; NH-CSS: Nethine- Harbison clinical scoring system; PCNV: Pathogenic copy number variation; SDS: Standard deviation score; SRS: Silver-Russell syndrome; \\ UPD(7)mat: Maternal uniparental disomy chromosome 7}

\begin{abstract}
Acknowledgements
We are grateful to all patients and their parents for their cooperation. We thank the following doctors for providing us with detailed clinical data and materials for molecular studies: Reiko Horikawa, Tomoki Kosho, Rika Kosaki, Keisuke Nagasaki, Koji Muroya, Hiroshi Suzumura, Tatsuhiko Urakami, Mitsuo Masuno, Yoko Miyoshi, Yosuke Ichihashi, Yuji Oto, Shiho Tamaura, Jun Mori, Hiroshi Yoshihashi, Sumito Dateki, Yoriko Watanabe, Takahito Wada, Noriko Kinoshita, Yoko Hiraki, Ken Nagaya, Yasuhiro Suzuki, Toshiro Yoshioka, Kanako Ishii, Kosuke Yamada, Tatenobu Goto, Shinji Hasagawa, Kei Izumi, Ken Fukuda, Chiho Tokorotani, Toju Tanaka, Shinsuke Ninomiya, Tatsuyuki Tanaka, Rei Nishimura, Shuntaro Morikawa, Yasuyuki Fukuhara, Kei Takasawa, Hideaki Yagasaki, Kayo Kunisada, Junko Saito, Chikahiko Numakura, Yohei Matsubara, Seiko Maeno, Takahiro Mochizuki, Mayu Yamamoto, Isamu Kamimaki, Yoshiyuki Kobayashi, Norioki Ono, Nobuyuki Murakami, Toshihiro Nakajima, Tomoki Kokeguchi, and Yoshihiro Aoki. We also thank Ms. Emma Barber for her editorial assistance with this paper.
\end{abstract}

\section{Funding}

This work was supported by Grants from the Japan Society for the Promotion of Science (JSPS) (15K15096), the National Center for Child Health and Development (28-6), the Japan Agency for Medical Research and Development (AMED) (16ek0109030h0003 and 16ek0109141 h0002), Takeda Science Foundation, and The Japanese Society for Pediatric Endocrinology Future Development Grant.

\section{Availability of data and materials}

Not applicable.

\section{Authors' contributions}

Molecular analysis was performed by TI, AN, TF, KY, SS, and KM. Detailed clinical data and materials for molecular studies were provided by SM, YM, $\mathrm{CH}, \mathrm{TH}, \mathrm{HN}, \mathrm{KT}, \mathrm{ZK}$, and TO. The study was designed and coordinated by MK. The paper was written by $\mathrm{TI}$ and $\mathrm{MK}$ and reviewed and edited by $\mathrm{AO}$ and MF. All the authors read and approved the final manuscript.

\section{Competing interests}

The authors declare that they have no competing interests.

\section{Consent for publication}

We obtained written informed consent from the patients or the patients' parents to publish patients' clinical and molecular information as well as facial photographs.

\section{Ethics approval and consent to participate}

This study was approved by the Institutional Review Board Committee at the National Center for Child Health and Development (committee's reference number: 518) and was performed after obtaining written informed consent to participate in this study from the patients or the patients' parents.

\section{Publisher's Note}

Springer Nature remains neutral with regard to jurisdictional claims in published maps and institutional affiliations.

\section{Author details}

'Department of Molecular Endocrinology, National Research Institute for Child Health and Development, 2-10-1, Okura Setagaya-ku, Tokyo 157-8535, Japan. ${ }^{2}$ Department of Pediatrics, The University of Tokyo Hospital, 7-3-1 Hongo, Bunkyo-ku, Tokyo 113-8655, Japan. ${ }^{3}$ Department of Pediatrics, Central Hospital, Aichi Human Service Center, 713-8 Kagiya-cho, Kasugai, Aichi 480-0392, Japan. ${ }^{4}$ Department of Pediatrics, The Japan Baptist Hospital, 47 Yamanomoto-cho, Kitashirakawa, Sakyo-ku, Kyoto 606-8273, Japan. ${ }^{5}$ Department of Pediatrics, Kyoto Prefectural University of Medicine, 465 Kajii-cho, Kamigyo-ku, Kyoto 602-8566, Japan. ${ }^{6}$ Tsumura Family Clinic, Kumi Shounika, 858-1 Watarihashi-cho, Izumo, Shimane 693-0004, Japan.

${ }^{7}$ Department of Pediatrics, Japanese Red Cross Kyoto Daiichi Hospital, 15-749 Honmachi Higashiyama-ku, Kyoto 605-0981, Japan. ${ }^{8}$ Department of Pediatrics, Hamamatsu University School of Medicine, 1-20-1 Handayama, Higashi-ku, Hamamatsu, Shizuoka 431-3192, Japan.

Received: 12 February 2017 Accepted: 1 May 2017

Published online: 15 May 2017

\section{References}

1. Wakeling EL, Brioude F, Lokulo-Sodipe O, O'Connell SM, Salem J, Bliek J, et al. Diagnosis and management of Silver-Russell syndrome: first international consensus statement. Nat Rev Endocrinol. 2017;13:105-24.

2. Lai KY, Skuse D, Stanhope R, Hindmarsh P. Cognitive abilities associated with the Silver-Russell syndrome. Arch Dis Child. 1994;71:490-6.

3. Price SM, Stanhope R, Garrett C, Preece MA, Trembath RC. The spectrum of Silver-Russell syndrome: a clinical and molecular genetic study and new diagnostic criteria. J Med Genet. 1999:36:837-42.

4. Netchine I, Rossignol S, Dufourg MN, Azzi S, Rousseau A, Perin L, et al. 11 p15 imprinting center region 1 loss of methylation is a common and specific cause of typical Russell-Silver syndrome: clinical scoring system and epigeneticphenotypic correlations. J Clin Endocrinol Metab. 2007:92:3148-54.

5. Bartholdi D, Krajewska-Walasek M, Ounap K, Gaspar H, Chrzanowska KH, Ilyana $\mathrm{H}$, et al. Epigenetic mutations of the imprinted IGF2-H19 domain in Silver-Russell syndrome (SRS): results from a large cohort of patients with SRS and SRS-like phenotypes. J Med Genet. 2009;46:192-7.

6. Bruce S, Hannula-Jouppi K, Puoskari M, Fransson I, Simola KO, LipsanenNyman M, et al. Submicroscopic genomic alterations in Silver-Russell syndrome and Silver-Russell-like patients. J Med Genet. 2010;47:816-22.

7. Dias RP, Nightingale P, Hardy C, Kirby G, Tee L, Price S, et al. Comparison of the clinical scoring systems in Silver-Russell syndrome and development of modified diagnostic criteria to guide molecular genetic testing. J Med Genet. 2013:50:635-9.

8. Azzi S, Salem J, Thibaud N, Chantot-Bastaraud S, Lieber E, Netchine I, et al. A prospective study validating a clinical scoring system and demonstrating phenotypical-genotypical correlations in Silver-Russell syndrome. J Med Genet. 2015;52:446-53.

9. Fuke $T$, Mizuno S, Nagai T, Hasegawa T, Horikawa R, Miyoshi $Y$, et al. Molecular and clinical studies in 138 Japanese patients with Silver-Russell syndrome. PLoS One. 2013:8:e60105.

10. Eggermann T, Meyer E, Obermann C, Heil I, Schüler H, Ranke MB, et al. Is maternal duplication of 11p15 associated with Silver-Russell syndrome? J Med Genet. 2005;42:e26. 
11. Spengler S, Schönherr N, Binder G, Wollmann HA, Fricke-Otto S Mühlenberg R, et al. Submicroscopic chromosomal imbalances in idiopathic Silver-Russell syndrome (SRS): the SRS phenotype overlaps with the 12q14 microdeletion syndrome. J Med Genet. 2010:47:356-60.

12. Spengler S, Begemann M, Ortiz Brüchle N, Baudis M, Denecke B, Kroisel PM, et al. Molecular karyotyping as a relevant diagnostic tool in children with growth retardation with Silver-Russell features. J Pediatr. 2012;161:933-42.

13. Sachwitz J, Meyer R, Fekete G, Spranger S, Matulevičienè A, Kučinskas V, et al. NSD1 duplication in Silver-Russell syndrome (SRS): molecular karyotyping in patients with SRS features. Clin Genet. 2017;91:73-8.

14. Kagami M, Matsubara K, Nakabayashi K, Nakamura A, Sano S, Okamura K, et al. Genome-wide multilocus imprinting disturbance analysis in Temple syndrome and Kagami-Ogata syndrome. Genet Med. 2017;19:476-82.

15. Zeschnigk M, Albrecht B, Buiting K, Kanber D, Eggermann T, Binder G, et al. IGF2/H19 hypomethylation in Silver-Russell syndrome and isolated hemihypoplasia. Eur J Hum Genet. 2008;16:328-34.

16. Wakeling EL, Amero SA, Alders M, Bliek J, Forsythe E, Kumar S, et al. Epigenotype-phenotype correlations in Silver-Russell syndrome. J Med Genet. 2010;47:760-8.

17. Russo S, Calzari L, Mussa A, Mainini E, Cassina M, Di Candia S, et al. A multimethod approach to the molecular diagnosis of overt and borderline 11p15.5 defects underlying Silver-Russell and Beckwith-Wiedemann syndromes. Clin Epigenetics. 2016;8:23.

18. Database of Genomic Variants. 2016. http://dgv.tcag.ca/dgv/app/home. Accessed 1 Feb 2017.

19. Battaglia A, Carey JC, South ST. Wolf-Hirschhorn syndrome: a review and update. Am J Med Genet C Semin Med Genet. 2015;169:216-23.

20. Melo JB, Estevinho A, Saraiva J, Ramos L, Carreira IM. Cutis Aplasia as a clinical hallmark for the syndrome associated with 19q13.11 deletion: the possible role for UBA2 gene. Mol Cytogenet. 2015;8:21.

21. Wu YQ, Sutton VR, Nickerson E, Lupski JR, Potocki L, Korenberg JR, et al. Delineation of the common critical region in Williams syndrome and clinical correlation of growth, heart defects, ethnicity, and parental origin. Am J Med Genet. 1998;78:82-9.

22. Trisomy 18 syndrome. In: Kenneth Lyons Jones, Marilyn Crandall Jones, Miguel del Campo. Smith's recognizable patterns of human malformation 7th ed. Philadelphia: Elsevier Saunders; 2013. p. 14-19.

23. Williams syndrome. In: Kenneth Lyons Jones, Marilyn Crandall Jones, Miguel del Campo. Smith's recognizable patterns of human malformation 7th ed. Philadelphia: Elsevier Saunders; 2013. p. 160-3.

24. Chauvel PJ, Moore CM, Haslam RH. Trisomy-18 mosaicism with features of Russel-Silver syndrome. Dev Med Child Neurol. 1975;17:220-4.

25. Tucker ME, Garringer HJ, Weaver DD. Phenotypic spectrum of mosaic trisomy 18: two new patients, a literature review, and counseling issues. Am J Med Genet A. 2007;143:505-17.

26. Adalat S, Bockenhauer D, Ledermann SE, Hennekam RC, Woolf AS. Renal malformations associated with mutations of developmental genes: messages from the clinic. Pediatr Nephrol. 2010:25:2247-55.

27. Venegas-Vega C, Nieto-Martínez K, Martínez-Herrera A, Gómez-Laguna L, Berumen J, Cervantes A, et al. 19q13.11 microdeletion concomitant with ins (2;19) (p25.3; 13.1q13.4) dn in a boy: potential role of UBA2 in the associated phenotype. Mol Cytogenet. 2014;7:61

28. Meyer E, Carss KJ, Rankin J, Nichols JM, Grozeva D, Joseph AP, et al. Mutations in the histone methyltransferase gene KMT2B cause complex early-onset dystonia. Nat Genet. 2017:49:223-37.

29. Ghanim M, Rossignol S, Delobel B, Irving M, Miller O, Devisme L, et al. Possible association between complex congenital heart defects and 11p15 hypomethylation in three patients with severe Silver-Russell syndrome. Am J Med Genet A. 2013;161:572-7.

30. Feuk L, Kalervo A, Lipsanen-Nyman M, Skaug J, Nakabayashi K, Finucane B, et al. Absence of a paternally inherited FOXP2 gene in developmental verbal dyspraxia. Am J Hum Genet. 2006;79:965-72.

\section{Submit your next manuscript to BioMed Central and we will help you at every step:}

- We accept pre-submission inquiries

- Our selector tool helps you to find the most relevant journal

- We provide round the clock customer support

- Convenient online submission

- Thorough peer review

- Inclusion in PubMed and all major indexing services

- Maximum visibility for your research

Submit your manuscript at www.biomedcentral.com/submit
C Biomed Central 